\title{
THE ACCURACY OF PLANTATION SURVEYS
}

\author{
By R. H. BLythe, Jr., B.Sc. \\ Sheffield Scientific School 1930, Yale Forest School, Assistant Silviculturist, \\ Lake States Forest Experiment Station
}

$\mathbb{W}$ ITH the advent of the C.C.C. camps and other relief labor organizations, the planting program of the Forest Service has been greatly expanded, particularly in the Lake States region. As a result, it has been necessary to develop methods of measuring the success and failure of the plantations in order to learn the effect of species, planting methods and site selection. The plantation survey has been designed to supply this information. A complete census or a 100 percent count of all planted trees is not practical and the examination of plantations must, therefore, be limited to a partial survey, based on some scheme which mechanically assures that a representative sample is examined. Since only a part of each plantation is covered, it becomes necessary to determine the reliability of the result obtained by such surveys. An analysis of the errors of a timber survey by Mudgett and Gevorkiantz (1) explains a method which is here made applicable to plantation surveys.

In order that comparisons between plantations may be made, it is necessary to determine the errors inherent in the estimates of survival and growth. Only by doing this can any differences between plantations be evaluated as real differences or merely fluctuations within the limits of sampling errors.

Determination of the errors of average height or diameter growth presents no unusual features, but in computing the error attached to the survival percent certain factors which have been overlooked in past work of this sort must be given proper consideration.

Since it is not feasible to examine every tree in a plantation, a systematic method of counting is employed. One of the best methods is indicated in the diagram (Figure 1). The observer follows the rows of planted trees; he counts twenty-five trees and then shifts to the next adjacent row to right or left and counts twenty-five more, and then shifts again in the same direction to the next row, proceeding across the entire area in this manner. Any number of such strips may be run across the plantation depending on the intensity of the survey. The purpose in shifting rows is to include the work of the different planters in the sample.

Survival in plantations is measured in terms of percentage. In the field the trees are classified as growing, living, failing or dead. The number of 


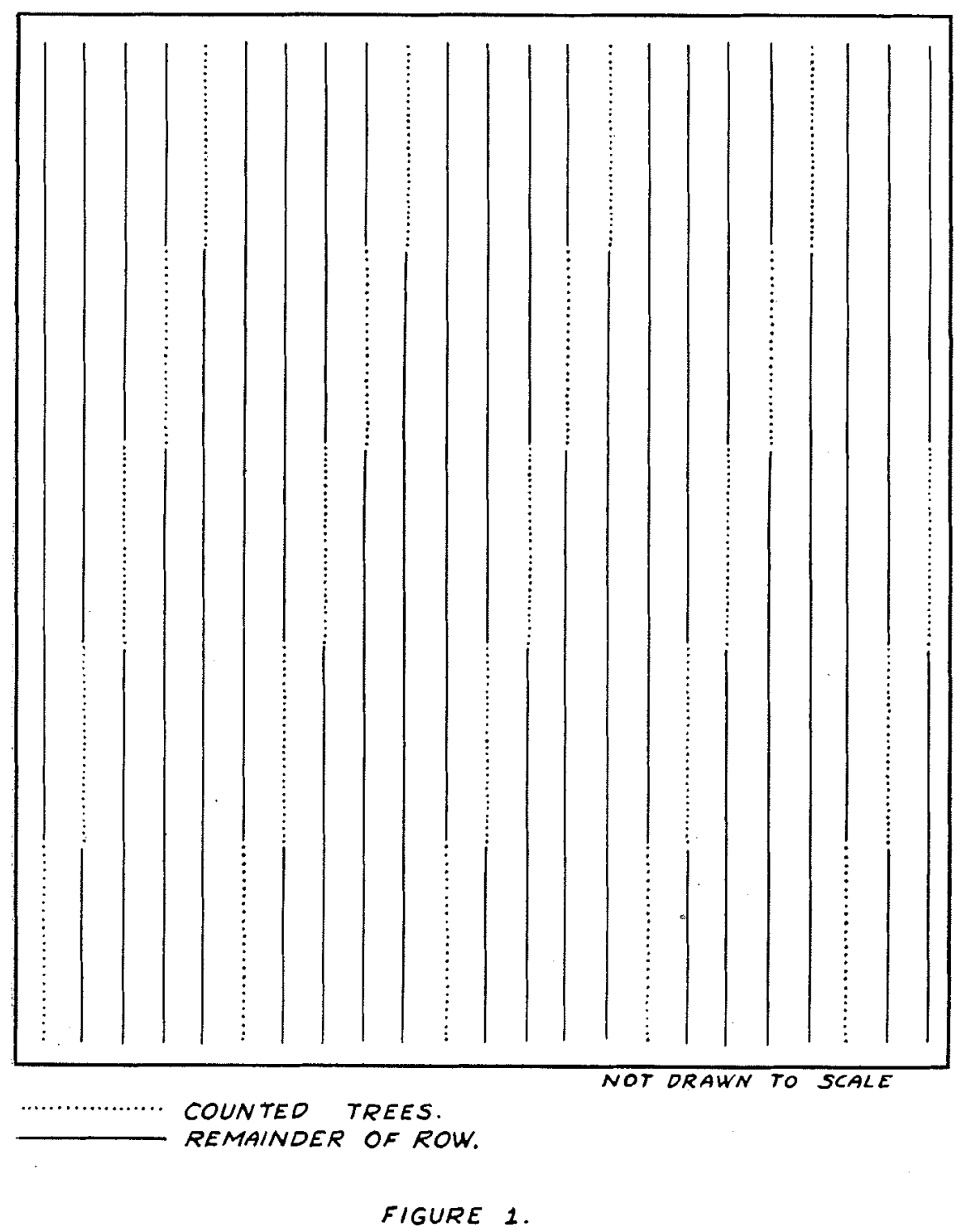


trees in the first two groups are added together and expressed as a percent of the total number in all four of the classes. This figure is denoted as the "survival percent."

In the language of statistics this process is called sampling for attributes, while the determination of the average height growth is termed sampling for variables. A classical case of sampling for attributes commonly discussed in statistical texts is the tossing of a coin and classification of the throws as heads or tails. The mathematical laws underlying this case have been worked out in detail and statistical methods for analyzing the results of studies involving sampling for attributes have been developed on the basis of these laws. However, certain assumptions (2) which underlie the coin tossing problem limit the application of the statistical technique to those cases which can meet the assumptions. All too often the technique has been applied to cases which superficially appear to be parallel with the classic examples, coin tossing or dice throwing, but which in reality are not. A rational consideration of many of these problems would immediately arouse suspicion as to the applicability even though no preliminary analysis of data were made.

'The fundamental difference between the coin tossing problem and plantation surveys can be explained by simple reasoning without recourse to statistical theory. Consider the case of twenty throws of a perfect coin; obviously "heads" will be expected to appear about ten times. If the twenty throws are repeated five times, it is not expected that ten heads will appear each time. Even if the coin is perfect, there will certainly be some variation in the number of times heads will appear. This is ascribed to "chance."

Likewise, it is evident that if the average survival of an entire plantation is 50 percent, it cannot be expected that a survival of 50 percent will be found in every small subdivision of that plantation. This variation is ascribable partly to "chance," but a portion of the variation is due to other factors and the addition of this variation is what makes the plantation survey problem entirely different from the coin tossing problem. The death of certain trees in a plantation is dependent upon a number of causes. Poor planting, inferior seedlings, unfavorable soil conditions, drought, insect or rabbit injury, heavy shade, and other factors are responsible for the death of individual trees in a plantation. Some of these factors will probably be present in about equal degree all over any single plantation; but others, particularly soil and brush cover, will vary locally. If it were possible to determine the boundary lines between areas within which each of these several factors is constant, then the variation in survival between subdivisions of these homogeneous areas would be due wholly to chance. Unfortunately, it is not possible to outline, in the field, areas throughout which these factors are constant. Most plantation surveys are run 
using the plantation as a unit of area. This may vary in size from 5 to 1000 or more acres. In large areas more variation in soil and topography will be encountered, and in a region of irregular surface formations the heterogeneity of the conditions affecting survival will be greater than in a sand plain region, for example.

\section{STATISTICAL TECHNIQUE}

The coin tossing problem will hereafter be referred to as "simple sampling," which is the statistical term for the general problem of which coin tossing is one example.

The average number of successes and the standard deviation of this average can easily be computed for simple sampling problems. The standard deviation of the proportion of successes is given by the formula:

$$
{ }^{\sigma} \mathrm{B}=\sqrt{ } \frac{\mathrm{pq}}{\mathrm{n}}
$$

Where: $p$. is the probability of a success (in the case of plantation surveys, $\mathrm{p}=$ proportion of trees that are alive).

$\mathrm{q}$ is the probability of a failure $(\mathrm{q}=1-\mathrm{p})$ (in the case of plantation surveys, $q=$ proportion of trees that are dead).

$\mathrm{n}$ is the number of events (in the case of plantation surveys, $\mathbf{n}$ is the number of trees examined)

and ${ }^{\sigma} \mathrm{B}$ is the required standard deviation.

The application of this formula to plantation surveys may be illustrated as follows:

Suppose the average survival percent as obtained by a count of 1500 trees to be 62 percent; therefore, $\mathrm{p}=.62$ and $\mathrm{q}=.38$; then from formula (1) it follows:

$$
{ }^{\sigma} \mathrm{B}=\sqrt{ } \frac{.62 \times .38}{1500}=\vee .000157=.0125
$$

This formula has been used in exactly this manner in expositions on the application of statistical methods to forestry problems but it will be shown that this formula tells only a part of the story and the smallest part at that.

The universe to be sampled must meet three conditions before the formulas of simple sampling can be applied without violating mathematical theory. These are:

(1) The universe must be made up of single events which are completely independent of each other. Applied to a plantation survey, this means that the survival of any individual tree must not be influenced by the survival or death 
of any other individual. In young plantations this requirement is probably always satisfied.

(2) There must be no essential difference between the localities from which the individuals of the sample are drawn. In the case of plantation surveys, this requirement need not be satisfied except when samples are taken from several plantations and then lumped into a single sample for purposes of determining the average survival and its standard deviation.

(3) The conditions which control the appearance of the observed character must be the same for every individual in every sample; that is, the universe must be homogeneous. For plantations, this means that the conditions affecting survival must be the same for every seedling in the plantation. Probably no plantation survey is able to meet this requirement. The conditions which are responsible for the survival of individual trees vary greatly within the boundaries of most plantations, or in statistical terms, the universe is heterogeneous. (It is conceivable that a small plantation might be located in an area where soil conditions, brush cover, etc.., would be essentially similar.)

This heterogeneity of the condition responsible for survival adds another source of variation to that due to simple sampling. The addition to this variation does not mean that the problem of determining the accuracy of survival percents cannot be solved, but it does mean that the formulas of simple sampling must be modified to take care of the new factor.

The statistical technique for handling cases of this nature is not new. It was developed ( 3 and 4 ) by the German mathematician, Lexis, around 1879, and is generally known as the Lexian theory. This theory is applicable to a great many practical problems which cannot be attacked by the method of simple sampling because of the rigid restrictions which must be adhered to in making the observations.

The necessity for using the Lexis theory in the case of plantation survival counts can be demonstrated by the analysis of the data from an actual survey made on the Superior National Forest.

In this plantation, 1750 trees were counted. In making the survey, the trees were tallied in twenty-five tree units, and the survival percent computed for each such unit. For purposes of statistical analysis, the 70 units of 25 trees each were apportioned into ten samples. In order that each of the ten samples might be representative of the entire plantation, and therefore as nearly alike as possible, each was made up of non-adjacent twenty-five tree units. Sample one, for example, consisted of units $1,11,21,31,41,51,61$, or seven of the 25 tree units; sample two of units $2,12,22$, etc. In this way ten samples of 175 trees each were synthesized, and each sample was made up of trees from all over the plantation. 
The survivals of the ten samples are listed in Table 1:

\begin{tabular}{rcc}
$\begin{array}{c}\text { Sample } \\
\text { No. }\end{array}$ & $\begin{array}{c}\text { No. of } \\
\text { Trees } \\
\text { Surviving }\end{array}$ & $\begin{array}{r}\text { Survival } \\
\text { percent }\end{array}$ \\
1 & 126 & 72.0 \\
2 & 127 & 72.6 \\
3 & 101 & 57.7 \\
4 & 110 & 62.9 \\
5 & 117 & 66.9 \\
6 & 115 & 65.7 \\
7 & 125 & 71.4 \\
8 & 122 & 69.7 \\
9 & 136 & 77.7 \\
10 & 125 & 71.4 \\
\hline Ave. & 120.4 & 68.8
\end{tabular}

The standard deviation of these averages by the formula applicable to simple sampling is:

$$
\sigma_{\mathrm{B}}=\vee \frac{(.688)(.312 \underline{)}}{175}=\vee .00122661=.0350
$$

If the standard deviation of these ten averages is worked out according to the usual method, applicable to the sampling of variables, the following result is obtained:

$$
\begin{aligned}
& { }^{\sigma} \mathrm{A}=\vee-\frac{\Sigma(X-M)^{2}}{\mathrm{n}} \\
& \sigma_{\mathrm{A}}=\vee .00289460=.0538
\end{aligned}
$$

Where: $X=$ the individual observations

$M=$ the mean of the individual observations

$\mathrm{n}=$ the number of observations

This is a considerably larger value than that given by the formula for simple sampling. The difference between the actual standard deviation $\left({ }^{\sigma} \mathrm{A}\right)$ of the ten means as just computed and the theoretical $\left({ }^{\sigma} \mathrm{B}\right)$ is due to the heterogeneity of the conditions affecting survival. The actual standard deviation $(\sigma \mathrm{A})$, as computed above, approaches the correct value for the error of the mean survival percent determined from a count of 175 trees.

However, 1750 trees in all have been measured and it is necessary to determine the standard error of a mean based on that number of trees, since greater accuracy naturally results from using the total number of observations. The problem then is to determine the error when the number of observations 
is stepped up from 175 to 1750 . The most accurate method would be to secure several more samples of 1750 observations from the same plantation and determine their standard deviation, as was done in the case of samples of 175 items. But then it would be desirable to know the error of the mean based on the new total. This process would thus go on ad infinitum.

For practical use, a simple method is available. The actual standard deviation of the means of 175 is made up of two factors - the variation due to the fluctuations of simple sampling, and the variation introduced by the heterogeneity of the survival. In order that these factors may be additive, it is necessary to express them as "variances", which are numerically equal to the square of the standard deviation.

The relationship between the actual variance and the other two variances is given by the following formula:

$$
\sigma_{\mathrm{A}^{2}}=\sigma \mathrm{B}^{2}+\sigma_{\mathrm{h}^{2}}
$$

The third term of the equation is the variance due to heterogeneity. Its numerical value is determined by subtraction:

$$
{ }^{\sigma} \mathrm{h}^{2}=\sigma^{\sigma} \mathrm{A}^{2}-{ }^{\sigma} \mathrm{B}^{2}
$$

substituting the values obtained in the example cited above:

$$
\begin{aligned}
\sigma_{\mathrm{h}^{2}}= & .00289460-.00122661 \\
& =.00166799
\end{aligned}
$$

This variance due to heterogeneity does not change with changes in the number of observations, and if enough data have been collected so that the factor, $h^{2}$, can be determined by splitting the total number of observations into a number of samples and calculating the actual standard deviation of the means of the several samples, then it is possible to estimate the error of a mean based on any number of observations.

Substituting equation (1) in equation (2)

$$
\sigma^{\mathrm{A}^{2}}=\frac{\mathrm{pq}}{\mathrm{n}}+{ }^{\sigma} \mathrm{h}
$$

All the factors on the right hand side of the equation are obtainable from the data: $p$ is the proportion of living trees in the total sample; and $q=1-p$ as before; $n$ is the total number of observations or the number of trees counted in each sample; and $\sigma^{2}$ is obtained in the manner explained above.

\section{PRACTICAL APPLICATION}

In using this method, several conditions should be observed. When dividing the total number of observations into the several samples, the aim should be to keep the number of observations in each sample as large as possible and 
yet divide the data into a sufficient number of samples so that the standard deviation of the means can be determined on a reasonably good basis. These two aims are mutually exclusive and therefore a compromise is necessary. Ordinarily, ten samples should be the minimum limit, but where the number of observations is small, it may be necessary to reduce the number of samples to around six.

If the formula for the variance of the means of a heterogeneous universe (Formula 3) is considered critically, it will at once be apparent that the portion of the variance contributed by the fluctuations of random sampling $\left({ }^{\sigma} \mathrm{B}^{2}\right)$ decreases as $\mathrm{n}$ increases, but that the variance due to the heterogeneity of conditions $\left({ }^{\sigma} \mathrm{h}^{2}\right)$ does not become smaller as the number of observations is made larger. This is the situation in the case of an unlimited universe.

However, plantations are limited universes; that is, the survival percent obtained from the survey is intended to apply solely to the individual plantation and not to an indefinitely large number of plantations having similar conditions. In a limited universe, an increase in the number of observations reduces the variance due to heterogeneity as well as that due to the fluctuations of random sampling.

As a concrete example, consider the case of a forty acre plantation with approximately one thousand planted trees per acre, or a total of 40,000 trees in the limited universe of this single plantation. Suppose 100 trees per acre are counted to determine survival. This is a ten percent sample. If the number of observations is stepped up to 400 trees per acre, the sample is forty percent of the total number, and naturally a more accurate estimate of survival percent would be expected. The effect of increasing the relative intensity of the survey is expressed by the following formula:

$$
{ }^{\sigma} \mathrm{A}^{2}=\left(\frac{\mathrm{pq}}{\mathrm{n}}+{ }^{\sigma} \mathrm{h}^{2}\right) \frac{\mathrm{W}-\mathrm{n}}{\mathrm{W}-1}
$$

where $W$ is the total number of events in the universe and $n$ is the number of observations. When $n$ is very small compared to $W$, the factor for the limited universe has but little effect on the error, but as $n$ approaches $W$, its effect becomes considerable. When $n$ equals $W$, the factor becomes zero and the error also zero.

The factor of heterogeneity should be worked out for each plantation but this is not always possible, as it is often desirable to determine the accuracy of surveys which have already been completed and for which the data are not in the best form for such an analysis.

A general figure for this factor has been determined for each of three National Forests in the Lake States. In order to determine this factor it was necessary to combine the surveys from a number of plantations and then 
divide the combination into ten sub-samples so that each sub-sample contained an equal number of trees from each plantation. In this way any differences in survival between plantations were eliminated in making up the sub-samples.

The values of the variance due to heterogeneity are given in Table 2 .

Table 2

Area

Huron Nat'l. Forest, Michigan

Heterogeneity

Factor $\left(h^{2}\right)$

.000143

.000707

.001668

Superior Nat'l. Forest, Minnesota

The heterogeneity on the Huron National Forest is the least, and the reason for this is not hard to find. The plantations analyzed on this National Forest were located on sand plains where conditions of soil, topography, and overhead cover are not widely different. Conditions on the Superior are in marked contrast, the topography is varied, soil conditions change rapidly and brush cover with its correlated factor, rabbit damage, is widely variable. This is reflected in the high value of the heterogeneity factor for this area.

\section{REFERENCES}

(1) Mudgett, Bruce D. and S. R. Gevorkiantz, 1934.

"Reliability of Forest Surveys," Journal of the American Statistical Association, 29. No. 187, September, 1934.

(2) Yule, G. U.

"An Introduction to the Theory of Statistics," London, 1919. Charles Griffin \& Co. Ltd.

(3) Rietz, Henry Lewis, 1929.

"Mathematical Statistics."

The Carus Mathematical Monographs No. 3. The Open Court Publishing Co., Chicago, Ill.

(4) Davis, Harold T., and W. F. C. Nelson, 1935.

"Elements of Statistics."

The Principia Press, Inc., Bloomington, Indiana. 